\title{
Liste des sigles
}

\section{Glossaire des institutions}

AEN : Agence pour l'énergie nucléaire de l'OCDE (NEA : Nuclear Energy Agency, OECD) AFCEN : Association française pour les règles de conception, de construction et de surveillance en exploitation des matériels des chaudières électronucléaires

AIEA : Agence internationale de l'énergie atomique, Vienne, Autriche (IAEA : International Atomic Energy Agency)

AISI : American Iron and steel Institute (Institut américain du fer et de l'acier)

ANCCLI : Association nationale des comités et commissions locales d'information

ANL : Argonne National Laboratory (Laboratoire national d'Argonne aux États-Unis)

AREVA : Concepteur et exploitant nucléaire français (transformé en Orano et Framatome)

ARILL : Association des retraités de l'Institut Laue-Langevin, France

ASME : American Society of Mechanical Engineers (désigne couramment les règles de conception et de construction établies par cette société américaine et utilisées par des concepteurs de réacteurs nucléaires [Westinghouse, etc.])

ASN : Autorité de sûreté nucléaire, France

AVN : Association Vinçotte-Nucléaire, Belgique

CCR : Centre commun de recherche, Commission européenne

CEA : Commissariat à l'énergie atomique et aux énergies alternatives, France

CERCA : Compagnie pour l'étude et la réalisation de combustibles atomiques, filiale d'AREVA, France

$\mathrm{Cl}$ : Commission d'information

CIPR : Commission internationale de protection radiologique

CIS : Commission interne de sécurité

$\mathrm{CLI}$ : Commission locale d'informations 
CNRS : Centre national de la recherche scientifique, France

CSIA : Commission de sûreté des installations atomiques

DAE : Département atomique indien

DEP : Direction des équipements sous pression, ASN

DOE : Department of Energy (Ministère de l'Énergie américain)

DSN : Département de sûreté nucléaire du CEA

DSND : délégué à la sûreté nucléaire et à la radioprotection pour les activités et installations intéressant la défense nationale française

EDF : Électricité de France

ENSREG : European Nuclear Safety Regulators Group (groupe consultatif d'experts indépendants, de la Commission européenne)

FzK : Forschungszentrum Karlsruhe (Institut de technologie de Karlsruhe, Allemagne)

GAAA : Groupement atomique alsacienne atlantique, France

GRS : Gesellschaft für Anlagen - und Reaktorsicherheit (société pour la sûreté des installations et des réacteurs nucléaires, Allemagne)

HCTISN : Haut Comité pour la transparence et l'information sur la sécurité nucléaire, France

KIT (ex-FzK, ex-KfK) : Karlsruhe Institut für Technology (Institut de technologie de Karlsruhe, Allemagne)

ILL : Institut Laue-Langevin, France

INL : Idaho National Laboratory (Laboratoire national de l'Idaho, États-Unis)

IPSN : Institut de protection et de sûreté nucléaire, France

IRSN : Institut de radioprotection et de sûreté nucléaire, France

LLB : Laboratoire Léon Brillouin (unité mixte de recherche [UMR] CEA/CNRS), France

LSTC : Livermore Software Technology Corporation, États-Unis

OCDE : Organisation de coopération et de développement économique (OECD : Organisation for Economic Co-operation and Development)

PNC (ex-JAEA) : Power reactor and Nuclear fuel development Corporation, Japon

SCK CEN : Studiecentrum voor Kernenergie - Centre d'étude de l'énergie nucléaire, Belgique

SCSIN : Service central de sûreté des installations nucléaires, France

SODERA : Société pour le développement de la recherche appliquée, France

WENRA : Western European Nuclear Regulators Association (Association des chefs des autorités de sûreté des pays de l'Europe de l'ouest)

\section{Glossaire technique}

ADS : Accelerator Driven System (réacteurs hybrides sous-critiques)

AGM : accidents graves maîtrisés

ALARA : As Low As Reasonably Achievable (aussi bas que raisonnablement possible) principe de radioprotection

ALIZÉ : nom donné à un réacteur nucléaire de recherche du CEA (arrêté définitivement) 
APOLLO : nom donné à un logiciel de simulation neutronique en $2 \mathrm{D}$, qui établit des bibliothèques multi paramétrées de sections efficaces

AQUILON : nom donné à un réacteur nucléaire de recherche du CEA (arrêté définitivement)

ARS : arrêt d'urgence sismique (réacteur à haut flux à Grenoble)

ASTEC : Accident Source Term Evaluation Code (logiciel de simulation pour l'évaluation des phénomènes physiques intervenant au cours d'un accident de fusion du cœur d'un réacteur à eau sous pression)

ASTER : Analyses des structures et thermomécanique pour des études et des recherches (logiciel de simulation)

ASTRID : Advanced Sodium Technological Reactor for Industrial Demonstration (projet de réacteur de démonstration à neutrons rapides et refroidi au sodium)

ATPu : Atelier de technologie du plutonium (installation fermée ayant eu pour activité principale la production de combustible « MOX » [mélange d'oxydes d'uranium appauvri et de plutonium] pour les réacteurs nucléaires)

ATWS : Anticipated Transients Without Scram (transitoires avec échec de l'arrêt automatique)

AZALÉE : nom donné à une table vibrante du CEA à Saclay

AZUR : Alliage Zirconium Uranium (réacteur nucléaire de recherche relevant de la défense nationale)

BCS : barres de commande et de sécurité

BDBA : Beyond Design Basis Accident (domaine des accidents dits hors dimensionnement)

$B E P$ : boucle à eau sous pression (réacteur CABRI)

BNCT : Boron Neutrons Capture Therapy (thérapie de capture de neutrons par le bore utilisée pour traiter des tumeurs)

BORAX : BOiling water ReActor eXperiment (réacteur d'expérimentations à eau bouillante, États-Unis)

BR2 : Belgium Reactor 2 (réacteur au Centre de recherche de Mol en Belgique)

BSM : bâtiment de stockage et manutention (installation MASURCA)

CABRI : nom donné à un réacteur d'essai du CEA à Cadarache permettant l'étude de situations accidentelles dans les réacteurs (REP, RNR)

CASHIMA : nom donné à un projet de recherche relatif aux « effets de site » en matière sismique

Cast3M : nom donné à un code de calcul par la méthode des éléments finis pour la mécanique des structures et des fluides

CATHARE : Code avancé de thermohydraulique pour l'étude des accidents de réacteurs à eau (logiciel de simulation dédié aux analyses de sûreté)

CDS : circuit de dégonflage sismique (réacteur à haut flux à Grenoble)

CDU : critère de défaillance unique

CEN : circuit de pompage d'eau dans la nappe phréatique (réacteur à haut flux à Grenoble) 
CERES : nom donné à un logiciel de simulation dédié au calcul d'impact radiologique d'un rejet en situation accidentelle

CES : circuit de refroidissement d'eau de secours (réacteur à haut flux à Grenoble)

CESAR : nom donné à un réacteur de recherche du CEA (arrêté définitivement) ou nom donné à un module de thermohydraulique du logiciel ASTEC

CFD : Computational Fluid Dynamics (mécanique des fluides numérique ou MFN)

$\mathrm{CIP}$ : Cabri International Programme (programme international consacré à l'étude du comportement des crayons de combustible nucléaire et de leur gainage en cas d'accident d'injection de réactivité dans les réacteurs à eau sous pression)

CPA : nom donné à un module du logiciel ASTEC

CRAB : circuit de refroidissement à l'arrêt de la barre de pilotage (réacteur à haut flux à Grenoble)

CRISTAL : nom donné à un formulaire co-développé par l'IRSN, le CEA et AREVA ayant pour objectif l'évaluation du risque de criticité dans toutes les installations nucléaires et les emballages de transport mettant en œuvre des matières fissiles

CRONOS : nom donné à un logiciel de simulation qui détermine le comportement neutronique d'un cœur de réacteur en 3D

CRP : Coordinated Research Project (projet de recherche coordonné)

CRU : circuit de refroidissement d'ultime secours (réacteur à haut flux à Grenoble)

CSS : commission des normes de sûreté (AIEA)

DBA : Design Basis Accident (domaine des accidents dits de dimensionnement)

DEC : Design Extension Conditions (domaine de conception étendu)

DIRAS : dossier d'information relatif à l'assainissement des structures

DISCO : Dispersion of simulated corium (installation expérimentale de KIT dédiée à l'aérocontamination/la dispersion de corium, utilisant des poudres inactives)

DOR : dossier d'orientation du réexamen (réexamens périodiques de sûreté)

DULCINEE : nom donné à un logiciel de simulation permettant de simuler la thermohydraulique dans un cœur de réacteur et la thermique du combustible

ECS : évaluations complémentaires de sûreté (menées à la suite de l'accident de Fukushima Daiichi)

EIP : équipement important pour la protection (des « intérêts », notion de la règlementation française)

EL2 : Eau Lourde 2 (réacteur de recherche du CEA dorénavant démantelé)

EL3 : Eau Lourde 3 (réacteur de recherche du CEA en cours de démantèlement)

EL4 : Eau Lourde 4 (centrale nucléaire de Brennilis, France)

EOLE : nom donné à un réacteur nucléaire de recherche du CEA

EPIC : établissement public à caractère industriel et commercial

EPR : European Pressurized Water Reactor (réacteur européen à eau sous pression)

EPS : études probabilistes de sûreté

ESPN : équipements sous pression nucléaires 
ETRR-2 : Egypt Test and Research Reactor Number two (réacteur nucléaire de recherche égyptien $n^{\circ} 2$ )

EUROPLEXUS : nom donné à un logiciel de simulation par éléments finis

FINAS : Fuel Incident Notification and Analysis System (système de notification et d'analyse des incidents de combustible géré par l'AIEA)

FLICA : nom donné à un logiciel de simulation qui calcule la thermohydraulique d'un cœur de réacteur lors de transitoires

FRM-II : Forschungsreaktor München II (réacteur de recherche implanté à Garching en Allemagne)

GAZAXI : nom donné à un logiciel dédié au calcul d'impact radiologique d'un rejet en situation accidentelle

GPD : Groupe permanent d'experts en charge de déchets

GPE : Groupes permanents d'experts

GPESPN : Groupe permanent d'experts en charge des équipements sous pression nucléaires

GPMED : Groupe permanent d'experts en charge de la radioprotection dans les applications médicales

GPR : Groupe permanent d'experts en charge des réacteurs nucléaires

GPRAD : Groupe permanent d'experts en charge de la radioprotection dans les applications industrielles et de recherche des rayonnements ionisants

GPT : Groupe permanent d'experts en charge des transports

GPU : Groupe permanent d'experts en charge des laboratoires et usines

GUS : groupe d'ultime secours (réacteur Jules Horowitz)

HARMONIE : nom donné à un réacteur nucléaire de recherche du CEA (dorénavant démantelé)

HEMERA : Highly Evolutionary Methods for Extensive Reactor Analyses (Méthodes hautement évolutives pour une analyse approfondie des réacteurs - chaîne de calcul qui permet la simulation thermohydraulique et neutronique de transitoires dans un réacteur nucléaire)

HFR : High Flux Reactor (réacteur à haut flux du Centre commun de recherche [CCR] de Petten, Pays-Bas)

HIFAR : High Flux Australian Reactor (réacteur à haut flux australien)

HTR : High Temperature Reactor (réacteurs à haute température)

IEA-R1 : Instituto de Energia Atômica-Reactor 1 (réacteur de recherche brésilien)

IGORR : International Group on Research Reactors (groupe d'experts internationaux sur les réacteurs de recherche)

INB : installation nucléaire de base

INES : International Nuclear Event Scale (échelle internationale des événements nucléaires développée par l'AIEA)

INSAG : International Nuclear Safety Group (groupe d'experts internationaux en sûreté nucléaire) 
INSARR : Integrated Safety Assessment of Research Reactors (Évaluation intégrée de la sûreté des réacteurs de recherche)

IODE : nom donné à un module du logiciel ASTEC

IRR1 : Israël Research Reactor-1 (réacteur de recherche $\mathrm{n}^{\circ} 1$ israélien)

IRS : International Reporting System for Operating Experience (système pour la déclaration d'incidents pour les réacteurs de puissance géré par l'AIEA)

IRSRR : Incident Reporting System for Research Reactor (système de déclaration des incidents pour les réacteurs de recherche géré par l'AIEA)

ISIS : nom donné à la maquette critique du réacteur nucléaire de recherche OSIRIS à Saclay

ISTP : International Source Term Program (programme de recherche international sur le « terme source »)

ITER : International Thermonuclear Experimental Reactor (réacteur thermonucléaire experimental international, en construction à Cadarache)

JEPP : jours équivalents à pleine puissance

LS-DYNA : nom donné à un logiciel de simulation par éléments finis de calcul dynamique de structures

LVR-15 : Light Water Reactor-15 (réacteur à eau légère de la République tchèque)

MARIUS : nom donné à un réacteur nucléaire de recherche du CEA (arrêté définitivement) MASURCA : maquette critique du CEA à Cadarache

MC3D : nom donné à un logiciel de thermohydraulique multiphasique 3D pour la simulation de l'interaction entre des matériaux fondus et un fluide réfrigérant

MCNP : Monte Carlo N-Particule transport code (logiciel de simulation en 3D du transport de particules fondé sur la méthode de Monte-Carlo)

MDT ou MDTE : manque de tension externe

MDTG : manque de tension généralisé

MELUSINE : nom donné à un réacteur nucléaire de recherche du CEA (dorénavant démantelé)

MINERVE : nom donné à un réacteur nucléaire de recherche du CEA à Cadarache MNSR : Miniature Neutron Source Reactor (réacteur miniature source de neutrons) MORET : logiciel de simulation qui résout l'équation du transport des neutrons à partir des méthodes Monte Carlo, principalement dédié aux études de criticité

MOX : Mixed Oxide Fuel (combustible mixte $\mathrm{UO}_{2}+\mathrm{PuO}_{2}$ )

MTR : Materials Testing Reactors (réacteurs d'irradiation technologique destinés principalement à des études et des tests de divers matériaux et combustibles nucléaires)

NRU : National Research Universal (réacteur de recherche des Chalk River Laboratories, Canada)

NRX : National Research eXperimental (réacteur de recherche des Chalk River Laboratories, Canada)

NSRR : Nuclear Safety Research Reactor (réacteur de recherche japonais utilisé pour des essais de sûreté) 
NUSSC : NUclear Safety Standards Committee (comité de l'AIEA sur les normes de sûreté des réacteurs nucléaires)

OLC : Operationnal Limits and Conditions (limites et conditions d'exploitation)

ONG : organisations non gouvernementales

OPAL : Open Pool Australian Lightwater (réacteur de recherche de type piscine ouverte à eau légère australien)

ORPHÉE : nom donné à un réacteur nucléaire de recherche du CEA à Saclay

OSIRIS : nom donné à un réacteur nucléaire de recherche du CEA à Saclay (arrêté définitivement).

OTHELLO : nom donné à une boucle expérimentale du réacteur OSIRIS

$\mathrm{PAl}$ : pièges à iode

PCm : pour cent mille

PCR : personne compétente en radioprotection

PCS : poste de contrôle et secours (réacteur à haut flux à Grenoble)

PEGASE : nom donné à un réacteur de recherche du CEA (arrêté définitivement)

PEGGY : nom donné à la maquette critique du réacteur de recherche PEGASE

$\mathrm{PF}$ : produits de fission

PGA : Peak Ground Acceleration (accélération à fréquence infinie)

PHEBUS : nom donné à un réacteur d'expérimentation du CEA à Cadarache

Phébus-PF : programme international de recherche consacré à l'étude du comportement des produits de fission en situations de fusion du cœur dans un réacteur à eau sous pression

PHENIX : nom donné à un prototype de réacteur électronucléaire (et d'expérimentations) du CEA à Marcoule, à neutrons rapides et utilisant du sodium liquide comme fluide caloporteur (actuellement en cours de démantèlement)

PIE : Postulated Initiating Events (événements initiateurs postulés)

PPI : plans particuliers d'intervention

PROSERPINE : nom donné à un réacteur de recherche du CEA (arrêté définitivement) PUI : plan d'urgence interne

RA-2 : nom donné à un réacteur de recherche argentin

RADIOSS : nom donné à un logiciel de simulation par éléments finis

RAPSODIE : nom donné à un réacteur expérimental à neutrons rapides et refroidi au sodium du CEA à Cadarache (arrêté définitivement)

RASSC : Radiation Safety Standards Committee (comité de l'AIEA sur les normes de sûreté radiologique)

RCC-CW : règles de conception et de construction du génie-civil des ilots nucléaires des réacteurs à eau sous pression ( $\mathrm{CW}$ comme civil work)

RCC-E : règles de conception et de construction des équipements électriques et de contrôle-commande des ilots nucléaires des réacteurs à eau sous pression

RCC-M : règles de conception et de construction pour des matériels mécaniques 
RCC-MRx : règles de conception et de construction pour les matériels mécaniques des structures à hautes températures et des réacteurs expérimentaux et à fusion

REP : réacteur à eau sous pression

RERTR : Reduced Enrichment for Research and Test Reactors (programme américain visant à réduire l'enrichissement pour les réacteurs de tests et de recherche)

RFS : règles fondamentales de sûreté

$R G E$ : règles générales d'exploitation

RGEP : rupture guillotine d'un élément particulier (notion utilisée dans l'analyse de sûreté du réacteur Jules Horowitz)

RGSE : règles générales de surveillance et d'entretien

RHF : réacteur à faut flux (réacteur nucléaire de recherche de l'Institut Laue-Langevin, à Grenoble)

RIA : Reactivity Injection Accident (accident d'insertion de réactivité)

RIB : retrait intempestif d'une barre de commande

$\mathrm{RJH}$ : réacteur Jules Horowitz (réacteur nucléaire de recherche du CEA actuellement en cours de construction)

RNR : réacteurs à neutrons rapides (refroidis au sodium)

RRDB : Research Reactor DataBase (base de données de l'AIEA relative aux réacteurs de recherche)

RSE-M : règles de surveillance en exploitation des matériels mécaniques (réacteurs à eau sous pression)

RSG-GAS : Reaktor Serba Guna - Gerrit Augustinus Siwabessy (réacteur de recherche indonésien)

RUS : réacteur universitaire de Strasbourg ou circuit de réfrigération de sauvegarde secondaire du RJH

SAFARI-1 : South African Fundamental Atomic Research Installation 1 (réacteur nucléaire de recherche sud-africain)

SCANAIR : logiciel de simulation du comportement thermomécanique des crayons de combustible des réacteurs à eau sous pression au cours de transitoires de puissance

SCARABEE : nom donné à un réacteur de recherche du CEA à Cadarache (dorénavant démantelé)

SIGMA : Seismic Ground Motion Assessment (programme d'évaluation des mouvements sismiques du sol)

SILOE : nom donné à un réacteur nucléaire de recherche du CEA à Grenoble (actuellement en cours de démantèlement)

SILOETTE : nom donné à un réacteur nucléaire de recherche à Grenoble (maquette critique du réacteur nucléaire SILOE) du CEA (dorénavant démantelé)

SIMMER : nom donné à un logiciel couplant neutronique et mécanique des fluides qui permet de simuler un accident de fusion de combustible dans un réacteur à neutrons rapides SIREX : Système d'instrumentation pour réacteurs expérimentaux

SL-1 : Stationary Low Power Reactor Number One (réacteur stationnaire de faible puissance $n^{\circ} 1$ américain, Idaho National Laboratories, Idaho) 
SLR : situations de limitation du risque (notion utilisée dans l'analyse de sûreté du réacteur Jules Horowitz)

SMHV : séisme maximal historiquement vraisemblable

SMS : séisme majoré de sécurité

SND : séisme « noyau dur »

SOFIA : Simulateur d'observation du fonctionnement incidentel et accidentel (simulateur du fonctionnement des réacteurs à eau sous pression utilisé par l'IRSN)

SPERT : Special Power Excursion Reactor Tests (réacteur d'essais américain dit d'excursion de puissance)

SSC : Structures, Systems and Components (structures, systèmes et composants)

TECDOC : TEChnical DOCuments (documents techniques, AIEA)

TECV : loi française relative à la transition énergétique et à la croissance verte $\mathrm{n}^{\circ} 2015$ 992 du 17 août 2015

THE : très haute efficacité

TRANSSC : TRANsport Safety Standards Committee (comité de l'AIEA sur les normes de sûreté des transports de matières radioactives)

TREAT : Transient Reactor Test Facility (réacteur de recherche développé par Idaho National Laboratories, Idaho Falls, États-Unis)

TRIDENT : nom donné à un logiciel de simulation

TRIGA : TRaining, Isotope, General Atomics (réacteur de recherche de type piscine développé par General Atomics, États-Unis)

TRIPOLI : TRIdimensionnel POLYcinétique (logiciel de simulation en 3D qui résout par la méthode de Monte-Carlo l'équation du transport couplé des neutrons et des photons) TSN : transparence et sécurité en matière nucléaire (loi française $n^{\circ} 2006-686$ du 13 juin 2006)

ULYSSE : nom donné à un réacteur nucléaire de recherche du CEA (dorénavant démantelé). Ce réacteur était de type argonaute, un modèle de réacteur universitaire développé par l'ANL, États-Unis (en anglais ARGONAUT signifie ARGOnne Nuclear Assembly for University Training)

UNGG : uranium naturel-graphite-gaz, filière de réacteurs français

VARMA : valeur résiduelle modélisée acceptable

VENUS : Vulcan Experimental Nuclear System (système nucléaire expérimental Vulcain, CCR de Mol en Belgique)

WASSC : WAste Safety Standards Committee (comité de l'AIEA sur les normes pour la sûreté de la gestion des déchets radioactifs)

ZEPHYR : Zero power Experimental PHYsics Reactor (réacteur de physique expérimental de puissance zéro en projet au CEA)

ZOÉ : Z comme zéro, O comme oxyde d'uranium et $\mathrm{E}$ comme eau lourde ou encore ZérO Énergie (est aussi appelé EL1 [EL comme eau lourde]). Premier réacteur de recherche français, installé au centre du CEA à Fontenay-aux-Roses 
